\title{
On the importance of intraindividual variation in nutritional research
}

\author{
O.F.A. Larsen ${ }^{1 *}$, E. Claassen ${ }^{1}$ and R.J. Brummer ${ }^{2}$ \\ ${ }^{1}$ Vrije Universiteit Amsterdam, Athena Institute, De Boelelaan 1085, 1081 HV, Amsterdam, the Netherlands; ${ }^{2}$ Nutrition- \\ Gut-Brain Interactions Research Centre, Faculty of Health and Medicine, School of Medical Sciences, Örebro University, \\ Fakultetsgatan 1, Örebro, 70182, Sweden; o.f.a.larsen@vu.nl
}

Received: 3 March 2020 / Accepted: 13 June 2020

(c) 2020 Wageningen Academic Publishers

OPEN ACCESS (1) (1)(2) RESEARCH ARTICLE

\begin{abstract}
Nutritional intervention studies, like those with pre- and probiotics, are often hampered by low effect sizes, reducing the power to demonstrate potential efficacy. Here, we perform computer simulations of a hypothetical clinical trial using such an intervention in order to elucidate determining factors that can be influenced in order to optimise the statistical power. Our simulations demonstrate that steering the study population towards a low intraindividual variation dramatically improves statistical power. A more than 10-fold decrease of number-to-treat could be reached. Also, a careful balancing between the number of subjects and measurements per subject, in combination with possible stratification of the subjects into responders and non-responders, based on inherent intraindividual variation, improves the likelihood to reach statistically significant results. Our results also show that traditional dogmas, with respect to clinical trials, i.e. aiming at low interindividual variation and a high number $(n)$ of study participants, should be re-evaluated in favour of reducing intraindividual variation. This reduction in intraindividual variation could be achieved by maintaining a steady lifestyle, including dietary habits among others, within the timeframe of the intervention study.
\end{abstract}

Keywords: probiotics, prebiotics, nutrition, clinical trial, statistics

\section{Introduction}

Prebiotics and probiotics have shown to be promising non-pharmaceutical candidates for the prevention and treatment of a variety of disorders (Sanders et al., 2019). Although promising results have been achieved (Panigrahi et al., 2017), providing clinical evidence for the use of these compounds remains, despite intense research efforts, hard to achieve (Kunk, 2019; McFarland and Goh, 2019). This lack of proven efficacy can be attributed to several factors. Some factors will be discussed briefly below for the case of probiotics, but analogous considerations could be made for prebiotics as well. In the case of probiotics, the mode of action is often strain dependent (Hill et al., 2014). This makes it inherently difficult (if not impossible) to objectively compare different probiotics in a meta-analysis. Moreover, the efficacy of probiotics has been shown to be affected by the product matrix as well, which (ultimo) requires to perform meta-analyses on complete products only (Flach et al., 2018). Second, probiotic action may be very well subject-dependent (i.e. personalised). Personalised probiotics can be defined as products that are specifically tailored (regarding strain(s), dose, matrix, etc.) to the (patho)physiological characteristics of the target subject, which are again, among others, defined by the individual intestinal ecosystem or immune response patterns. This implies that a specific probiotic agent may be efficacious for a sub-group of the target-population only (Zmora et al., 2018). Hence, a precision (personalised) design of probiotic interventional strategies rather than a 'one-size-fits-all' approach should be favoured (Larsen et al., 2019). Third, the effect sizes (i.e. the magnitudes of the clinical effects) of nutritional interventions are generally inherently low compared to that of pharmaceutical compounds (De Vos et al., 2006). Hence, if aiming to beneficially affect the level of some (health-associated) parameter by pre- and/ or probiotic administration, the small bio-functional effect of the interventional product must overrule both intraand interindividual variations of this parameter in the clinical trial study population, in order to reach statistical 
significance and hence biological/clinical relevance. Therefore, strategies to reach statistical significance may focus on reducing intraindividual and/or interindividual variation, as well as increasing the study population $(n)$ or the number of measurements per subject. The $P$-level is ultimately determined by the number of sampled subjects, the number of measurements per subject and the aforementioned fluctuations. Besides inventorying and possibly stratifying the subjects based on these fluctuations, one should also consider whether it is better to do more measurements per subject on a relatively small number of subjects, or to do less measurements per subject on a relatively large sample of subjects. Recently, the limitations of (randomised controlled) clinical trials using probiotics have been discussed qualitatively (Zeilstra et al., 2018). Here, we apply a quantitative approach by modelling a hypothetical clinical trial with sequential layout studying a (pre- or) probiotic intervention aimed at improving a specific physiological functionality, like increasing butyrate production or enhancing gut epithelial integrity, which can be represented by some biomarker $X$. We model this trial with respect to the number of subjects (sample size), the number of measurements per subject and intra- and interindividual variation, respectively. The results can be used to discuss and determine the optimal design of nutritional clinical intervention studies in general and specifically that of pre- and probiotics, in order to achieve clinical evidence, as well as to provide information on and improve identification of responders versus non-responders.

\section{Materials and methods}

We modelled a hypothetical sequential clinical trial which aims to significantly change the value (in this case: an uplift) of a health-related biomarker $X$ using a pre- or probiotic intervention.

\section{Simulation of the population of subjects and sample of subjects}

First, we simulated a hypothetical population representing the 'real' target-population parameters, i.e. the population average of $X$, designated as $\mu$, and the corresponding standard deviation $\sigma$. This population was modelled by 10.000 subjects. The value of $X$ was Gaussian distributed over all subjects, mimicking interindividual variations. To simulate fluctuations of $X$ over time for each subject, the 'real' average value of $X$ for each individual subject $i$, designated as $\mu_{\imath}$ was dressed using a Gaussian curve having a 'real' standard deviation $\sigma_{i}$. This set of $\sigma_{i}$ for all subjects was also Gaussian distributed, with mean $\bar{\sigma}$ and standard deviation $\sigma_{\bar{\sigma}}$. The average $\mu_{i}$ and corresponding standard deviation $\sigma_{i}$ for each subject were obtained from a set of Gaussian distributed values, $\left\{X_{i}\right\}$, mimicking the different values of $X_{i}$ at different timepoints due to intraindividual variations. In our simulations, we used 10,000 different timepoints. This leads to an initial population matrix consisting of 10,000 subjects times 10,000 timepoints (measurements per subject).

Subsequently, the pre- or probiotic intervention was simulated by $\Delta$, causing an offset in the $\mu_{i}$ for all subjects. The values of $\Delta$ were also Gaussian distributed having a standard deviation $\sigma_{\Delta}$, hence each subject has its own (personalised) $\Delta_{\mathrm{i}}$. This offset creates a second population matrix (population data after intervention), again consisting of 10,000 subjects times 10,000 timepoints. Note that although the average offset is set as a positive number, some $\Delta_{\mathrm{i}}$ are negative values because of its Gaussian character (mimicking, in this case, a decline in health-status upon pre- or probiotics administration).

A random subject sample with predefined size was drawn (without replacement) from the population of subjects. For each subject drawn, a predefined number of timepoints (measurements) was drawn at random (without replacement) from the set of timepoints before pre- or probiotic administration, $\left\{X_{i, \text { before }}\right\}$. Subsequently, the same predefined number of timepoints was also drawn at random (without replacement) from the set of timepoints after pre- or probiotics administration, $\left\{X_{i, \text { after }}\right\}$. As such, we can mimic a defined number of measurements performed on $X$ for each subject during the clinical trial, before and after pre- or probiotics administration, to determine both averages $\overline{X_{l, \text { before }}}$ and $\overline{X_{l, \text { after }}}$, estimating the real $\sigma_{i, \text { before }}$ and $\sigma_{i, \text { after }}$, respectively.

\section{Paired $t$-test}

First, for each subject $i$ the difference of the mean values of biomarker $X$ was calculated using:

$d_{i}=\overline{X_{l, \text { after }}}-\overline{X_{l, \text { before }}}$

In which $\overline{X_{l, a f t e r}}$ being the mean of $\left\{X_{i, \text { after }}\right\}$, the set of measurements after administration of pre- or probiotics, and $\overline{X_{l, \text { before }}}$ the mean of $\left\{X_{i, \text { before }}\right\}$, the set of measurements before administration of pre- or probiotics. Subsequently, we can calculate $\bar{D}$, simply being the sample mean of the set of differences $\left\{d_{i}\right\}$, using:

$\bar{D}=\frac{1}{n} \sum_{i=1}^{n} d_{i}$

With $n$ being the number of sample subject pairs. The variance belonging to a single value of $d_{i}$ is given by:

$\operatorname{VAR}\left(d_{i}\right)=\operatorname{VAR}\left(\left\{X_{i, a f t e r}\right\}-\left\{X_{i, \text { before }}\right\}\right)=$
$\frac{\operatorname{VAR}\left(\left\{X_{i, a f t e r}\right\}\right)+\operatorname{VAR}\left(\left\{X_{i, \text { before }}\right\}\right)-2 \rho \sqrt{\operatorname{VAR}\left(\left\{X_{i, \text { before }}\right\}\right)} \sqrt{\operatorname{VAR}\left(\left\{X_{i, a f t e r}\right\}\right)}}{n_{m}}$ 
(Devore and Berk, 2012), with $n_{m}$ being the number of (paired) measurements and $\rho$ being the Pearson correlation coefficient. Subsequently, we calculate $\operatorname{VAR}\left(\left\{d_{i}\right\}\right)$, being the variance of the set of $d_{i}$, as the sum of the interindividual variance and intraindividual variance:

$$
\operatorname{VAR}\left(\left\{d_{i}\right\}\right)=\operatorname{VAR}\left(\left\{d_{i}\right\}\right)_{\text {inter }}+\operatorname{VAR}\left(\left\{d_{i}\right\}\right)_{\text {intra }}
$$

With:

$\operatorname{VAR}\left(\left\{d_{i}\right\}\right)_{\text {inter }}=\frac{1}{n} \sum_{i=1}^{n}\left(d_{i}-\bar{D}\right)^{2}$

And (Loève, 2017):

$\operatorname{VAR}\left(\left\{d_{i}\right\}\right)_{\text {intra }}=\frac{1}{n^{2}} \sum_{i=1}^{n} \operatorname{VAR}\left(d_{i}\right)$

The standard deviation $S_{D}$ of $\left\{d_{i}\right\}$ is, obviously:

$S_{D}=\sqrt{\operatorname{VAR}\left(\left\{d_{i}\right\}\right)}$

Now, the paired $t$-value can be calculated according to Devore and Berk (2012):

$t=\frac{\bar{D}}{S_{D} / \sqrt{n}}$

As we assumed an uplift in the value of the biomarker caused by the pre- or probiotic, we used a one-sided paired $t$-test. Normal Q-Q plots of $\left\{d_{i}\right\}$ were constructed to assess legitimacy of a paired $t$-test. All simulations were performed using R statistical software (R Core Team, 2019).

\section{Results}

Initially, we simulated two cases. In the first case, the interindividual variation was much larger than the relatively small intraindividual variation. In the second case, the interindividual variation was much smaller than the relatively large intraindividual variation. The parameters used for both cases are given in Table 1 . The results for both simulations were plotted in heatmaps, showing the $P$-values of the paired $t$-test as a function of both the subject sample size (i.e. the number of participants $n$ ) and the number of measurements performed in each subject (note again that this number gives the number of measurements for either before or after administration of the pre- or probiotics, and hence the full set of measurements per subject is two times this number). Heatmaps are shown in Figure 1A and Figure $1 \mathrm{~B}$ for case 1 and case 2, respectively. We define statistical significance as $P \leq 0.01$ (for this case the cells in the heatmaps are indicated in green). Note that for small sample sizes, Gaussian character of the difference data was rather poor, hence, a paired $t$-test was not fully legitimate. However, in order to keep consistent with the other data, a paired $t$-test, rather than a non-parametric test was performed.

Next, we executed two simulation-experiments using the same conditions as for case 1 and 2, respectively, in which we ordered the sample subjects according to the size of $\sigma_{i}$ (hence, intraindividual variation), after drawing the sample. Subsequently, we removed the $25 \%$ of subjects owing the highest $\sigma_{i}$, before performing the paired $t$-test as function of the number of measurements. For the construction of the heatmap corresponding to case 1, we took sample sizes from 4 up to 20 subjects. After taking $75 \%$ of the subjects every time, we end up with sample sizes ranging from 3 till 15 subjects. The result of this simulation is shown in Figure $1 \mathrm{C}$. For the construction of the heatmap corresponding to case 2, we took sample sizes of 20 up to 200 subjects, identical to the simulation just described for case 2 . After including $75 \%$ of the subjects every time, we end up with sample-sizes ranging from 15 till 150 subjects. The result of this simulation is shown in Figure 1D.

Table 1. Parameters used for the simulation of the two cases. See text for details.

$\begin{array}{lrrl}\text { Parameter } & \text { Case } 1 & \text { Case } 2 & \text { Explanation of parameter } \\ N & 10,000 & 10,000 & \text { Size population of subjects } \\ \mu & 500 & 500 & \text { Population average of } X \\ \sigma & 100 & 10 & \text { Population standard deviation of } X \text { (interindividual variation) } \\ M & 10,000 & 10,000 & \text { Number of time points simulated for each subject } \\ \bar{\sigma} & 10 & 100 & \text { Mean of the standard deviations } \sigma_{i} \text { of the individual subjects }\left(\sigma_{i} \text { due to intraindividual variation) }\right. \\ \sigma_{\bar{\sigma}} & 2 & 20 & \text { Standard deviation belonging to the Gaussian distribution of } \sigma_{i} \\ \Delta & 10 & 10 & \text { Mean effect pre- or probiotic intervention on } X \\ \sigma_{\Delta} & 10 & 10 & \text { Standard deviation belonging to Gaussian distribution of } \Delta_{i} \text { values }\end{array}$

${ }^{1}$ The total number of time points is two times this number, being the number of timepoints before pre- or probiotic administration and the same number of time points after pre- or probiotic administration. 

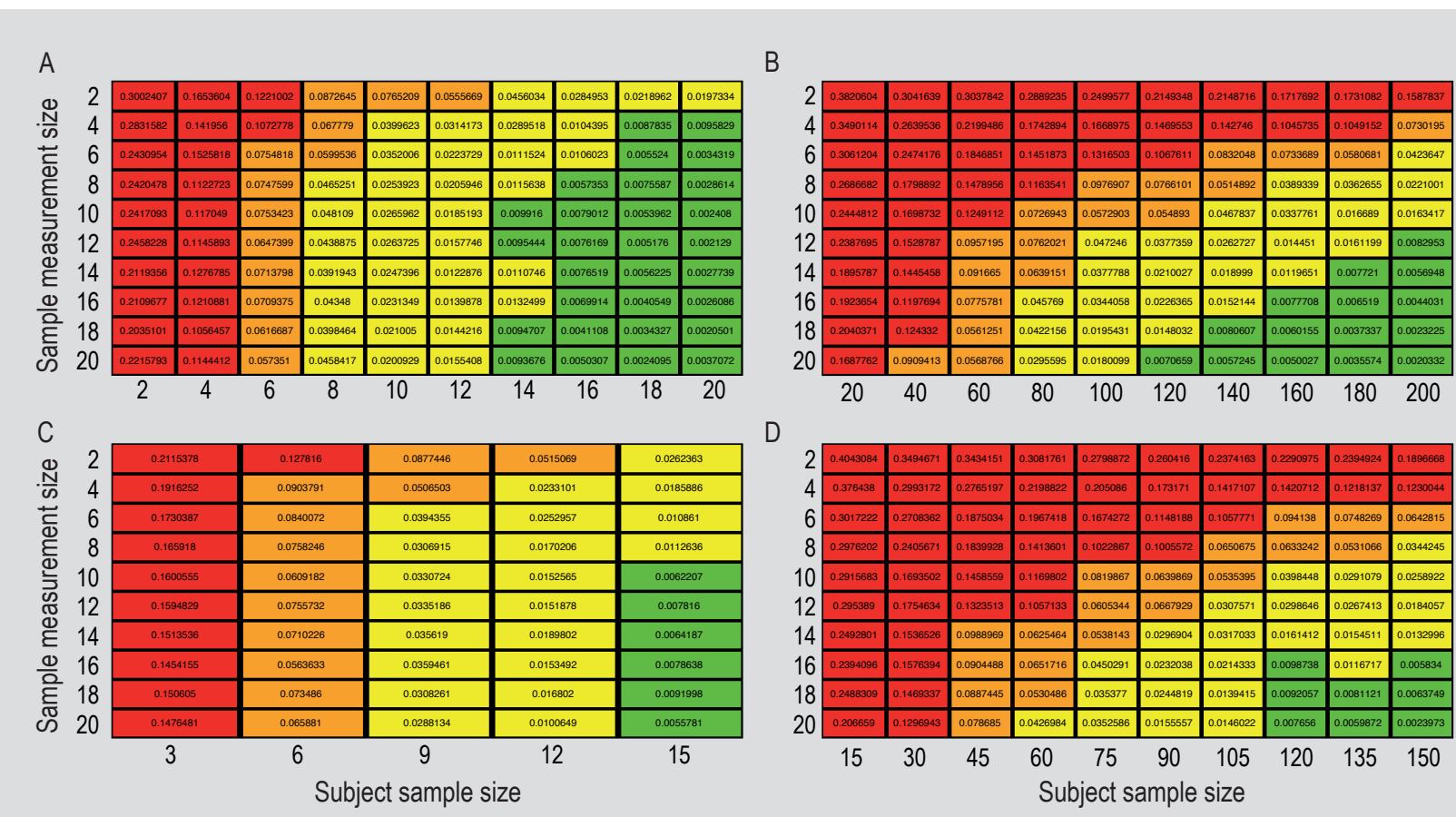

Figure 1. Heatmaps displaying the $P$-values of the paired $t$-test (rounded at 6 digits) as function of subject sample size and number of measurements per subject. Number of iterations to reduce statistical noise: 250 . Colour-coding for $P$-values: green: $P \leq 0.01$, orange: $0.01<P \leq 0.05$, yellow: $0.05<P \leq 0.1$, red: $P>0.1$ (see text for details). (A) The interindividual variation is much larger than the intraindividual variation (Case 1). (B) The intraindividual variation is much larger than the interindividual variation (Case 2). (C) Case 1, in which the subjects were ordered according to the size of initial intraindividual variation (ordering from highest to lowest sigma value), and the $25 \%$ of subjects owing the highest sigma values were removed before performing the paired $t$-test. Note: the sample sizes shown at the $x$-axis correspond to the sizes obtained after removing the $25 \%$ of subjects owing the highest $\sigma_{i \cdot}(\mathrm{D})$ analogous to $(\mathrm{C})$, but now for Case 2.

\section{Discussion}

Figure 1A shows that within the realm of performing maximally 20 measurements on 20 subjects, utilising the parameter values as defined for 'case 1' in Table 1, four different regimes considering $P$-value can be distinguished (as indicated by the different colours). Significant results (defined as $P \leq 0.01$ ) can be expected at sample subject sizes starting from $\sim 14$ persons (green coloured cells). The number of measurements needed to obtain significance decreases upon increasing the number of sampled subjects, ranging from $\sim 10$ (at $\sim 14$ subjects) down to only $\sim 4$ measurements if including $\sim 20$ subjects. If one would relax the significance level to values of $P \leq 0.05$, significant results are expected to be obtained already starting from $\sim 8$ subjects (yellow coded area). Clearly, performing the trial with 2-6 subjects may result in (very) insignificant outcomes (red and orange areas, respectively). Figure 1B, utilising the parameter values as defined for 'case 2' in Table 1 , shows a similar pattern as that for Figure 1A, albeit much higher numbers of subjects (up to 200) are needed in order to obtain statistically significant outcomes, if the number of measurements is limited to 20. Furthermore, the different significance areas in Figure 1B seem to be more along the diagonal compared to Figure 1A. In other words, in Figure $1 \mathrm{~B}$ the significance areas 'stretch out' more pronounced to lower number of subjects upon increasing the number of measurements, as compared to Figure $1 \mathrm{~A}$. Figure $1 \mathrm{C}$ and Figure 1D show the results of case 1 and 2, respectively, by paired $t$-test, after removing $25 \%$ of the sample subject population owing the highest $\sigma_{i}$. For these panels all four different significance areas are also present, and the areas for case 2 (Figure 1D) seem to be more diagonal as well, compared to case 1 (Figure 1C).

Applying these specific simulated cases, one requires substantially more subjects and measurements in order to reach significance using a paired $t$-test in case of high intraindividual variation, compared to the case of high interindividual variation. Nevertheless, in clinical practice a trial is often designed on basis of two traditional dogmas based on pharmaceutical interventions; (1) aiming at a homogeneous study population (subjects sample), i.e. the differences between mean values of $X$ between the subjects is as small as possible (low $\sigma$ ), and (2) including as many subjects $(n)$ as possible. However, the cases in this paper show that it may be better to aim for a low intraindividual variation instead. Hence, one should not only focus 
on minimising the variation of the clinical outcome parameter(s) between the subjects, but rather aim at keeping factors causing intraindividual variations as low as possible. As such, relatively few participants and measurements per subject are needed to reach statistical significance due to high stability of the outcome parameter(s) over time (low intraindividual variation), despite relatively large differences in biological characteristics and dietary intake that may exist between the subjects (causing large interindividual variation). The study regimen aiming at lowering the intraindividual variation should be designed in a way that it does not substantially impair the feasibility of the study, compliance of the participants and dietary variation.

In case 1 and case 2 one can 'compensate' a decrease in the number of subjects by increasing the number of measurements per subject. For example, in case 1, significance may be reached performing a trial with $\sim 20$ subjects and only $\sim 4$ measurements (i.e. 4 measurements before and 4 after pre- or probiotic administration), displaying a $P$-value of $\sim 0.0096$ (Figure 1A). If the number of subjects is limited to, for example, $\sim 16$ subjects, one can still reach significance by using $\sim 8$ measurements $(P$-value $\sim 0.0057$, Figure 1A). Similar arguments hold for Figure 1B displaying case 2 . Interestingly, this effect seems to be more pronounced for case 2 as reflected by the areas positioned more diagonally as compared to case 1 . This implies that one can, in this case of relatively high intraindividual variation, reduce the number of participants at the expense of increasing the number of measurements per subject. Hence, in the case of relatively large intraindividual variation, there seems to be more flexibility in choosing whether to include more subjects performing few measurements or to include less subjects but performing more measurements per subject. Nevertheless, the absolute numbers of measurements and subjects are much lower in case 1, which therefore seems to be the preferable option from an ethical, time, and costs-perspective.

Intraindividual variation may not be solely caused by fluctuating exogenous factors but may also be due to the subject's own inherent physiology (endogenous). In the latter case, initiatives to reduce the variation of exogenous factors, like dietary intake, may be of limited value. Hence, including a selection or stratification of subjects into potential responders and non-responders, based upon the level of intraindividual variation $\left(\sigma_{i}\right)$, at the design phase of the clinical trial, might be an effective way to reduce the number of participants and measurements necessary to achieve sufficient statistical power. This preselection is a logistic and methodological factor that should be implemented in the layout of the clinical trial and as such should be submitted to the ethical committee for approval as well. The consequences of such a stratification are exemplified by Figures $1 \mathrm{C}$ and 1D. These figures show the results for removing $25 \%$ of the subjects with largest intraindividual variance from the initial sample population in case 1 and 2, respectively. In the case of relatively low intraindividual variation (case 1), little to no effect is observed. For example, in Figure 1 the gross total number of measurements needed to obtain significance is $\sim 14$ subjects times $\sim 10$ measurements corresponding to $\sim 140$ measurements in total. In Figure 1C, we obtain very similar numbers at $\sim 15$ subjects times $\sim 10$ measurements corresponding to $\sim 150$ measurements gross in total. These differences are close to what can be considering statistical noise. Of course, intrinsically, the intraindividual variation in case 1 is generally low, hence, these results are not unexpected. A minor improvement based on individual variances may be well counterbalanced by the fact that the total variance inversely scales with the number of subjects (intra- and interindividual variance scale with respectively $1 / n^{2}$ and $1 / n$, see also Equations 5 and 6 ).

In case 2, characterised by high intraindividual variation, improvement can indeed be achieved by removing $25 \%$ of the sample population with the highest intraindividual variation before performing the paired $t$-test. Consequently, in Figure 1B one can still obtain significance $(P \sim 0.0071)$ at $\sim 120$ subjects and $\sim 20$ measurements resulting in a gross number of measurements of 2,400. However, after removing the worst $25 \%$ of $\sigma_{i}$ significance ( $P \sim 0.0099$, see Figure $\left.1 D\right)$ is obtained with 120 subjects and only 16 measurements per subject equalling a gross number of measurements of 1,920; i.e. a $20 \%$ reduction in the number of measurements. Again, one should be aware of the fact that by removing eligible participants having 'unfavourable' variances, the number of subjects $(n)$ is also reduced, partly counterbalancing the improvements made in variance (intra- and interindividual variances scale with respectively $1 / n^{2}$ and $\left.1 / n\right)$. Hence, careful balancing is needed. All the heatmaps displayed in this paper are the averages of many iterations. Because both subject sample size and measurement sample size are randomly taken out of the original population, the $P$-values are highly prone to statistical noise. This noise is smoothed by averaging 250 times per heatmap. Consequently, in a real-life experiment, it is very well possible that statistical significance is not reached although a large subject sample size and measurement size was chosen. Nevertheless, these simulations clearly show that a balanced choice between number of subjects and the number of measurements per subject, in combination with a possible selection or stratification of the subjects regarding potential responders and non-responders aiming for low intraindividual variation, may improve the odds for a successful outcome of a nutritional intervention study. Moreover, a substantial reduction in the gross number of measurements can be achieved if the experimental conditions can 'shift' from one characterised by high intraindividual variation towards one by low intraindividual variation This is clearly demonstrated by comparing Figure $1 \mathrm{~A}$ and $1 \mathrm{~B}$. Interestingly, these simulation results also give insight in potential applications 
'the other way around': by obtaining statistically significant outcomes one can extract subject-data regarding the intraand interindividual variation of the sampled population.

Our simulations show that a careful selection of subjects within its multidimensional parameter-space is essential for increasing the odds to obtain statistically significant results. Recent findings indeed suggest that the results obtained from such a carefully controlled trial setting may be transferable to the generally healthy population (Westerman et al., 2018). As such, computer simulations based on initially obtained target-population data has shown to be an indispensable tool. In Figure 2 we show, as an illustration, two simulation-results displaying the parameter spaces resulting in $P \leq 0.01$. The yellow dots display values of $P \leq 0.01$ as function of sample population size, sample measurement size and the mean standard deviation of the individual subjects $(\bar{\sigma})$, respectively. The other target-population parameters belonging to the blue dots are rather 'constraint' $\left(\mu=500, \sigma=100, \sigma_{\bar{\sigma}}=10, \Delta=5\right.$, $\left.\sigma_{\Delta}=10\right)$, resulting in a relatively small parameter space allowing $P \leq 0.01$. For the second simulation, which results are shown in blue (again, displaying values of $P \leq 0.01$ ), a much larger flexibility regarding the three parameters (as displayed on the axes) is allowed in order to expect significant results. The larger flexibility results from a more 'favourable' set of the remaining parameters of the targetpopulation $\left(\mu=500, \sigma=10, \sigma_{\bar{\sigma}}=1, \Delta=10, \sigma_{\Delta}=10\right.$, hence smaller $\sigma$ and $\sigma_{\bar{\sigma}}$ and larger $\Delta$ in comparison to the simulation resulting in the yellow dots). In other words, given this set of fixed target-population parameters, one has relatively large flexibility with respect to sample population size, sample measurement size and the mean standard deviation of the individual subjects $(\bar{\sigma})$. Please also note that the blue dots coincide with the yellow dots. Hence, at all coordinates displaying yellow dots, blue dots are also present (but not displayed to warrant clarity of the figure). Of course, the simulations can also be performed by varying other parameters and fixing the remaining parameters of the 8-dimensional parameter space. In Figure 2, the sphere provides a rough indication of the outer shell of the 'allowed' parameter space for the simulation with relaxed parameters: if one is capable to steer the parameters displayed on the axes within this space one increases the odds for statistically significant results.

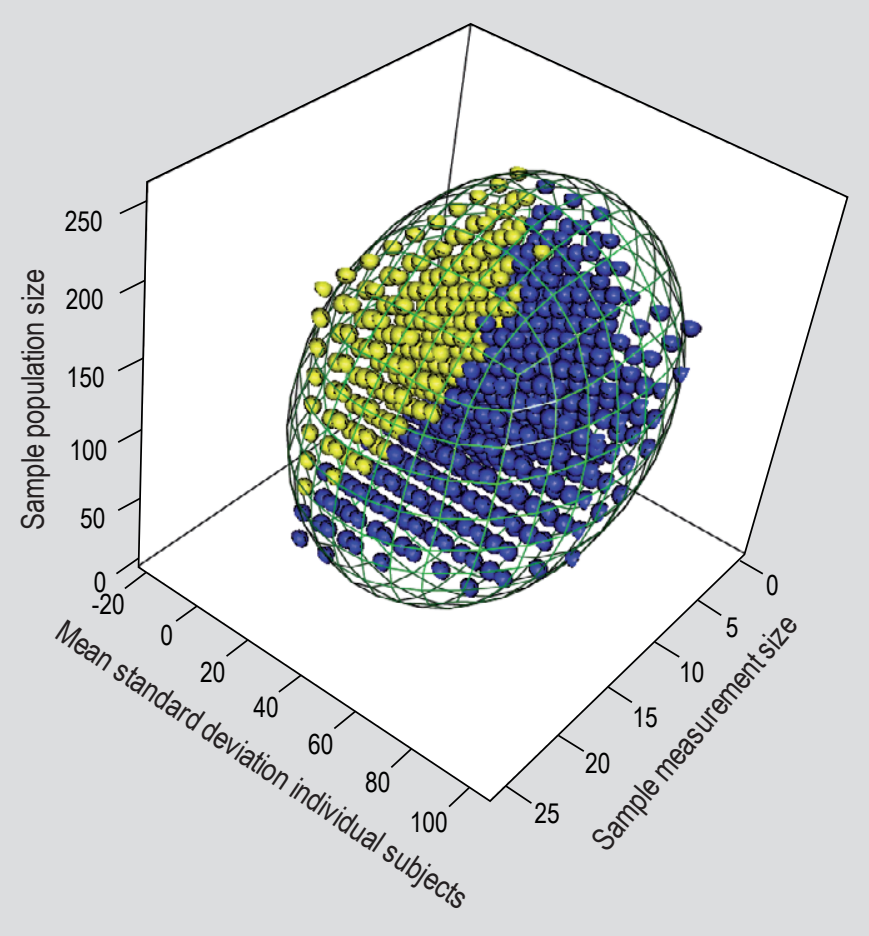

Figure 2. Simulation results showing the $P$-values with $P \leq 0.01$ for two different situations as function of the sample population size, the sample measurement size, and the mean standard deviation of the individual subjects. The blue dots result from relatively more favourable target-population parameters, whereas the yellow dots result from more unfavourable ones. Please note that the blue dots overlap with the yellow dots; at all yellow dots, blue dots are also present representing $P \leq 0.01$. Number of iterations to reduce statistical noise: 100 . The sphere roughly indicates the favourable parameter space for the blue dots: outside this space the odds are lower to obtain statistically significant results. See text for details. 


\section{Conclusions}

This paper shows that the power of a clinical trial can substantially be improved by carefully balancing between the number of measurements performed on each subject, total number of subjects participating and a possible selection or stratification of subjects with regard to potential responders and non-responders, based on the assessment of intraindividual variation of the principle study outcome parameter. A remarkable improvement can be achieved by focusing on a study population characterised by an overall small intraindividual variation rather than an overall small interindividual variation. This can be done by scrutinous trial-management minimising possible temporal fluctuations in exogenous lifestyle factors like dietary habits, physical exercise, mental stress, sleep, but also the use of prescribed and over-the-counter pharmaceuticals. According to our simulations, a 10-fold reduction of the required number of subjects to obtain statistically sound results could be achieved. This is beneficial from a time and financial resources perspective, but also counterfeits possible ethical objections to perform a clinical intervention study because of unfavourable burden/benefit ratio.

\section{Conflicts of interest}

O.L. is also Senior Manager Science at Yakult Nederland B.V. E.C. consults for most probiotic companies but has no competing interests with the current study. R.J.B. is also advisor to Christian Hansen.

\section{References}

De Vos, W.M., Castenmiller, J.J., Hamer, R.J. and Brummer, R.J.M., 2006. Nutridynamics - studying the dynamics of food components in products and in the consumer. Current Opinion in Biotechnology 17: 217-225. https://doi.org/10.1016/j.copbio.2006.02.008

Devore, J.L. and Berk, K.N., 2012. Modern mathematical statistics with applications, $2^{\text {nd }}$ edition. Springer, New York, NY, USA. https://doi. org/10.1007/978-1-4614-0391-3

Flach, J., Van der Waal, M.B., Van den Nieuwboer, M., Claassen, E. and Larsen, O.F.A., 2018. The underexposed role of food matrices in probiotic products: reviewing the relationship between carrier matrices and product parameters. Critical Reviews in Food Science and Nutrition 58: 2570-2584. https://doi.org/10.1080/10408398.2 017.1334624
Hill, C., Guarner, F., Reid, G., Gibson, G.R., Merenstein, D.J., Pot, B., Morelli, L., Canani, R.B., Flint, H.J., Salminen, S., Calder, P.C. and Sanders, M.E., 2014. Expert consensus document: Tshe International Scientific Association for Probiotics and Prebiotics consensus statement on the scope and appropriate use of the term probiotic. Nature Reviews Gastroenterology \& Hepatology 11: 506-514. https:// doi.org/10.1038/nrgastro.2014.66

Kunk, D., 2019. Probiotics: elixir or empty promise?. The Lancet Gastroenterology and Hepatology 4: 81. https://doi.org/10.1016/ S2468-1253(18)30415-1

Larsen, O.F.A., Koning, A.H., Van der Spek, P.J. and Claassen, E., 2019. Towards a rational design of faecal transplant analogues. Scientific Reports 9: 5558. https://doi.org/10.1038/s41598-019-42043-x

Loève, M., 2017. Probability theory, $3^{\text {rd }}$ edition. Dover Publications, Mineola, NY, USA.

McFarland, L.V. and Goh, S., 2019. Are probiotics and prebiotics effective in the prevention of travellers' diarrhea: a systematic review and meta-analysis. Travel Medicine and Infectious Disease 27: 1119. https://doi.org/10.1016/j.tmaid.2018.09.007

Panigrahi, P., Parida, S., Nanda, N.C., Satpathy, R., Pradhan, L., Chandel, D.S., Baccaglini, L., Mohapatra, A., Mohapatra, S.S., Misra, P.R., Chaudhry, R., Chen, H.H., Johnson, J.A., Morris, J.G., Paneth, N. and Gewolb, I.H., 2017. A randomized synbiotic trial to prevent sepsis among infants in rural India. Nature 548: 407-412. https:// doi.org/10.1038/nature23480

R Core Team, 2019. R: a language and environment for statistical computing. R Foundation, Vienna, Austria.

Sanders, M.E., Merenstein, D.J., Reid, G., Gibson, G.R. and Rastall, R.A., 2019. Probiotics and prebiotics in intestinal health and disease: from biology to the clinic. Nature Reviews - Gastroenterology and Hepatology 16: 605-616. https://doi.org/10.1038/s41575-019-0173-3 Westerman, K., Reaver, A., Roy, C., Ploch, M., Sharoni, E., Nogal, B., Sinclair, D.A., Katz, D.L., Blumberg, J.B. and Blander, G., 2018. Longitudinal analysis of biomarker data from a personalized nutrition platform in healthy subjects. Scientific Reports 8: 14685. https://doi.org/10.1038/s41598-018-33008-7

Zeilstra, D., Younes, J.A., Brummer, R.J. and Kleerebezem, M., 2018. Perspective: fundamental limitations of the randomized controlled trial method in nutritional research: the example of probiotics. Advances in Nutrition 9: 561-571. https://doi.org/10.1093/advances/ nmy046

Zmora, N., Zilberman-Schapira, G., Suez, J., Mor, U., Dori-Bachash, M., Bashiardes, S., Kotler, E., Zur, M., Regev-Lehavi, D., Brik, R.B.Z., Federici, S., Cohen, Y., Linevsky, R., Rothschild, D., Moor, A.E., Ben-Moshe, S., Harmelin, A., Itzkovitz, S., Maharshak, N., Shibolet, O., Shapiro, H., Pevsner-Fischer, M., Sharon, I., Halpern, Z., Segal, E. and Elinav, E., 2018. Personalized gut mucosal colonization resistance to empiric probiotics is associated with unique host and microbiome features. Cell 174: 1388-1405. https://doi.org/10.1016/j. cell.2018.08.041 
